\title{
CFD Simulation of a Shell and Tube Heat Exchanger
}

\author{
Mehmet Turgay Pamuk \\ Bahçeşehir University \\ Beşiktaş, Istanbul / Turkey \\ mehmetturgay.pamuk@eng.bau.edu.tr
}

\begin{abstract}
In this numerical work, CFD simulation of a Shell and Tube Heat Exchanger is performed using the commercial software ANSYS Fluent ${ }^{\odot}$. The CFD model is made to grab all the physical phenomena such as heat transfer rating, temperature, velocity and pressure distributions within the computational domain. A sufficiently high detailed geometry and fine mesh characteristics are adopted taking into account the computation resources and time, yet satisfactory enough to show that the numerical model can be validated using the results obtained from the calculations based on the heat transfer formulas, correlations, tables and graphs. This gives researches working in Heat Exchanger area the opportunity to design their systems using CFD, without depending on a prototype that needs to be tested before the actual product is marketed. Normally, a new type of heat exchanger with specific shell and tube dimensions and number of tubes has to be manufactured using general a heat exchanger calculation approach that will most of the time require the revision of the preliminary design.
\end{abstract}

Keywords: Heat Transfer, Heat Exchangers, CFD

\section{Introduction}

Heat Exchangers are essential equipment used in a wide area of applications ranging from HVAC to chemical processes, from power generation facilities to many sectors in industrial manufacturing. As the name suggests, it helps heat move from one medium to another. Radiator of a car helps most of heat generated by combustion and friction be carried to incoming air through the grills in front of a car. A pasteurizer in a dairy products manufacturing facility that heats up milk to a certain degree, then suddenly cools it down to kill bacteria, has also heat exchangers. However, not all these countless applications use the same type of heat exchangers. Depending on the required heat rate, cooled or heated media or physical limitations, they may be as simple as a concentric tube type heat exchanger, or as complicated as a plate type heat exchanger with a very high area-to-volume ratio. Among many types of heat exchangers, the shell and tube type is very common due to their very relatively simple structure, high heat exchange rate and sturdiness. It consists of a shell that houses a bundle of tubes and two caps covering the ends of the shell. Inside the tubes is one fluid, mostly the cooling agent such as water and outside the tubes is the fluid that needs to be cooled, such as oil, condensing steam etc. There are some previous studies on shell and heat exchangers in literature covering a wide range of diverse aspects. Hajabdollahi et al. [1] presented a comparative study for optimization of shell and tube heat exchangers and indicate that a noticeable improvement in the total cost is possible. For the cost optimization nine design parameters in the case of shell and tube heat exchanger were considered: inner and outer diameter of the tube, number of tube, number of tube pass, the ratio of tube pitch to tube diameter, tube arrangement, and baffle spacing ratio as well as baffle cut ratio. Wang et al. [2] investigated the methods for enhancing the heat transfer of a shell-and-tube heat exchanger. They found the methods to improve the capacity of heat exchanger through the installation of sealers in the shell-side, however at a cost in elevated pressure loss, although the benefit obtained from the capacity increase is much higher. They concluded that the sealers can be used in new heat exchangers or retrofitted into existing installations. The potential gain could be of benefit to many industries using shell-and-tube heat exchangers. Parikshit et al. [3] proposed a model in their paper to predict the shell side pressure drop of a shell and tube heat exchanger using the concept of Finite Element Method. Their model produced friction factors for pressure loss agreeable with those found in the literature. Vera-García et al. [4] proposed in their paper a simplified model for the study of shell-and-tubes heat exchangers. They performed several experiments for a shell and tube condenser and an evaporator used in refrigeration systems to validate their model and conclude that the proposed model constitutes a practical tool which can be easily used by designers, making it unnecessary to know the internal geometry of shell-andtubes HXs in order to obtain their behavior in a full installation. Wang et al. [5] investigated the thermodynamics 
performances for tube banks in crossflow and for the shell sides of shell-and-tube heat exchangers and analyzed the relation of fluid flow and heat transfer between them. Three types of STHXs with different structure baffles were selected to investigate the thermo-hydraulic performances for tube bundles in crossflow in the shell sides. Study on characteristics of heat transfer and fluid flow for tube banks in crossflow combining with the characteristics for tube bundles in the STHXs was carried out. Their results indicated that the incline degree of tube does not lead to obvious change on characteristics of fluid flow and heat transfer for fluid flowing across tube banks. Fettaka et al. [6] presented in their paper a multi-objective optimization of the heat transfer area and pumping power of a shell and tube heat exchanger to provide the designer with multiple Pareto-optimal solutions which capture the trade-off between the two objectives. They considered nine decision variables: tube layout pattern, number of tube passes, baffle spacing, baffle cut, tube-to-baffle diametrical clearance, shell-to baffle diametrical clearance, tube length, tube outer diameter, and tube wall thickness. Their results indicated that one can achieve a lower value of the heat transfer area and the pumping power as compared to the previously published values. Costa and Queiroz [7] presented in their paper a study about the design optimization of shell-and-tube heat exchangers. The formulated problem consists of the minimization of the thermal surface area for a certain service, involving discrete decision variables. The obtained results illustrate the capacity of their proposed approach to direct the optimization towards more effective designs, considering important limitations usually ignored in the literature. Raja et al. [8] presents in their paper an investigation of manyobjective (four-objective) optimization of shell and tube heat exchangers. They formed the many-objective optimization problem by considering maximization of effectiveness and minimization of total cost, pressure drop and number of entropy generation units of heat exchanger. Six design variables which include geometric parameters are considered for optimization. They found that the many objective approach leads to more desirable design of shell and tube heat exchangers as compared to multi-objective approaches. Haitao et al. [9] established a shell-side heat transfer model was to reveal the effect of tube bundle arrangement for flooded shell-tube evaporator, and validated it experimentally. Their simulation results agreed well with the experimental data with a deviation less than $15 \%$. They proposed an optimization method for uneven configuration of the horizontal and vertical tube spaces and obtained the optimized tube spaces. According to their findings, the performance of the optimized heat exchanger showed over $10 \%$ higher heat transfer capacity than the original one. Abda et al. [10] investigated the effect of shell diameter and tube length on heat transfer coefficient and pressure drop for shell side with both triangular and square pitches. They concluded that as shell diameter increases the heat transfer coefficient and pressure drop increases and the pull through head with triangular pitch can be the best choice to increase heat transfer coefficient. Zhou et al. [11] proposed an accurate and simplified model for predicting temperature distribution in the shell-and-tube heat exchanger on the basis of the differential theory. According to the baffle arrangement and tube passes, they divided the heat exchanger into a number of elements with tube side current in series and shell side current in parallel and noted that the proposed model can be successfully used for all shell-and-tube heat exchangers with straight tube or U-tube types.

\section{Theory}

Convection, one of the three modes of heat transfer suggests that heat is transported from a higher temperature medium to a lower temperature one, one of the media being a fluid, as a linear function of temperature difference between these two media. The proportionality constant called "heat transfer coefficient" is dependent upon many factors such as flow velocity and thermo-physical properties of the fluid medium, and the surface conditions and physical orientation of the solid (Eq. 1).

$$
\boldsymbol{q}^{\prime \prime}=h \Delta T
$$

Above equation gives the value for heat transfer rate per unit area, i.e. $\mathrm{W} / \mathrm{m}^{2}$. However, a heat transfer engineer mostly deals with the total heat transfer rate, i.e. $\dot{\boldsymbol{Q}}=\boldsymbol{q}^{\prime \prime} A$. Unfortunately, $\boldsymbol{q}^{\prime \prime}$ is not a simple figure to find out as it is a function of the length of the conduits most of the time, unless it is indicated as being constant. Therefore, for practical reasons, a more general approach is adopted (Eq.2): 


$$
\dot{Q}=U A \Delta T
$$

Here, $U$ is an overall heat transfer coefficient to include all the media, materials, surfaces and thermal resistances (Eq.3). R representing the thermal resistance such that $\dot{\boldsymbol{Q}}=\Delta \mathrm{T} / \mathrm{R}$

$$
R=\frac{1}{U A}=\frac{1}{h_{i} A_{i}}+R_{t}+R_{f}+\frac{1}{h_{o} A_{o}}
$$

where $R_{t}$ is the thermal resistance of the tube material and $R_{f}$ is the thermal resistance due to fouling. In the present analysis, they both have been neglected as the inner tube is copper, a high thermal conductivity material, and tubes are new. The most questionable part here in Eq. 2 is the temperature difference, $\Delta T$, as it varies along the heat exchanging surfaces, thus it is not a single value. However, differential analysis helps define a mean value for temperature difference $\Delta T_{m}$, or $L M T D$, logarithmic mean temperature difference as

$$
\Delta T_{m}=\frac{\Delta \boldsymbol{T}_{1}-\Delta \boldsymbol{T}_{2}}{\ln \left[\frac{\left.\Delta \boldsymbol{T}_{1}\right]}{\Delta \boldsymbol{T}_{2}}\right]}
$$

where $\Delta T_{1}$ and $\Delta T_{2}$ are the temperature differences of flow media at two ends of the heat exchanger. However, logarithmic mean temperature cannot be calculated unless all the temperatures are known which is most of the time not the case. There are two types of heat exchanger problems: the one where one is given the requirement for heating/cooling and a design for a brand new heat exchanger is initiated, and the other where one already has a heat exchanger at hand and needs to find out whether it serves a specific heat transfer need. Whichever the type of problem it is, one has to go through a detailed series of calculations, referring to various diagrams and tables and come up with a result that may substantially deviate from the reality due to the nature of experimental heat transfer. Deviation in the surplus side means overdesign, thus spending more money than necessary. On the other hand, under-design costs a lot more as it most of the time means sacrificing some of the equipment capacity, and maybe shutting down the plant. Using a CFD model will not only help define the geometry better, but also all the characteristics of the heat exchanger to be designed can be visualized beforehand so that the time spent and resources allocated will be minimal. The current study fits in the second type of heat exchanger analysis which is called the effectiveness method [12]. The numerical model for a shell and tube heat exchanger is validated using this method given for this type of heat exchangers. In this method, a definition for the maximum heat transfer possible is made as:

$$
\dot{\boldsymbol{Q}}_{\max }=C_{\min }\left(T_{h, i n}-T_{c, i n}\right)
$$

where $C_{\min }$ is the minimum of $\dot{\boldsymbol{m}}_{h} c_{h}$ and $\dot{\boldsymbol{m}}_{c} c_{c}$. Additionally, another definition, number of thermal units, $N T U=U A_{s} / C_{\min }$ is made in order to calculate the effectiveness $\epsilon$ of a shell and tube pipe heat exchanger (one-shell pass; $2,4, \ldots$ tube passes) such that $\dot{\boldsymbol{Q}}=\boldsymbol{\epsilon} \dot{\boldsymbol{Q}}_{\max }$ :

$$
\epsilon=2\left\{1+c+\sqrt{1+c^{2}} \frac{1+\exp \left[-N T U \sqrt{1+c^{2}}\right]}{1-\exp \left[-N T U \sqrt{1+c^{2}}\right]}\right\}^{-1}
$$

where $c=C_{\min } / C_{\max } . A_{s}$ is simply the outer surface area of the tubes. However, determining $U$, thus $h_{i}$ and $h_{o}$ requires many steps of calculation. Flow inside the pipes is laminar for the specified flow rate, however it is not developed hydrodynamically or thermally. Therefore Eq. 7 is used for the entry region:

$$
N u=3.66+\frac{0.065\left(\frac{d_{i}}{L}\right) \operatorname{RePr}}{1+0.04\left[\left(\frac{d_{i}}{L}\right) \operatorname{RePr}\right]^{\frac{2}{3}}}
$$

where $N u=h d_{i} / k_{f}$. and $k_{f}$ is the thermal conductivity of the fluid calculated at film temperature. 
Nusselt number calculation for the flow domain through the staggered array of tubes is performed using Eq. 8a and $8 b[13]:$

$$
\begin{gathered}
N u_{d}=1.04 \operatorname{Re}^{0.4} \operatorname{Pr}^{0.36}\left(\frac{P r}{P r_{s}}\right)^{0.25}, \text { for } 0<R e<100 \\
N u_{d}=0.71 \operatorname{Re}^{0.5} \operatorname{Pr}^{0.36}\left(\frac{P r}{P r_{s}}\right)^{0.25}, \text { for } 100<R e<1000
\end{gathered}
$$

where $R e=V d / v$ and $P r_{s}$ is the Prandtl number measured at the surface temperature. These correlations are valid for tube banks of $N>16$ and $0.7<P r<500$ and may have a deviation of $15 \%$. For tube banks less 16 , it is necessary to multiply the $N u_{\mathrm{d}}$ above by a correction factor $F$ which is 0.98 for the case in hand. In order to utilize Eq. 8, first of all the velocity need be defined as shown in Eq. 9:

$$
V_{\text {max }}=\frac{S_{T}}{2\left(S_{D}-d_{o}\right)} V
$$

with $S_{\boldsymbol{D}}=\sqrt{\boldsymbol{S}_{\boldsymbol{L}}^{2}+\left(\frac{\boldsymbol{S}_{\boldsymbol{T}}}{2}\right)^{2}}$ provided $S_{D}<\left(S_{T}+d_{o}\right) / 2$, where the definitions for $S_{T}, S_{D}$ and $S_{L}$ are shown in Fig. 1 where $V$ is the incoming fluid velocity.

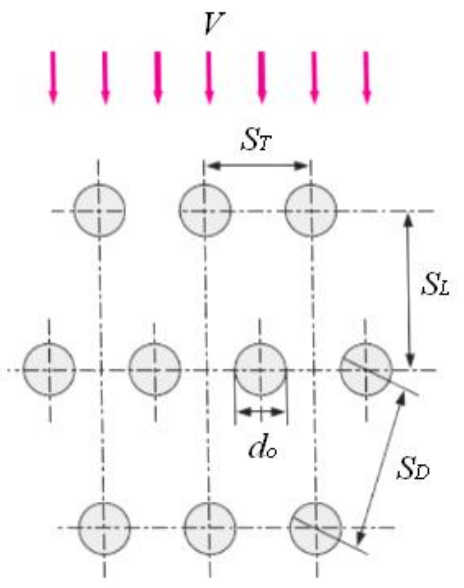

Fig. 1: Staggered array of tubes inside the heat exchanger.

As for the pressure drop, $\Delta P=N_{L} f X \frac{\rho V_{\max }^{2}}{2}$, the graph shown in Fig. 2 is used [14]:

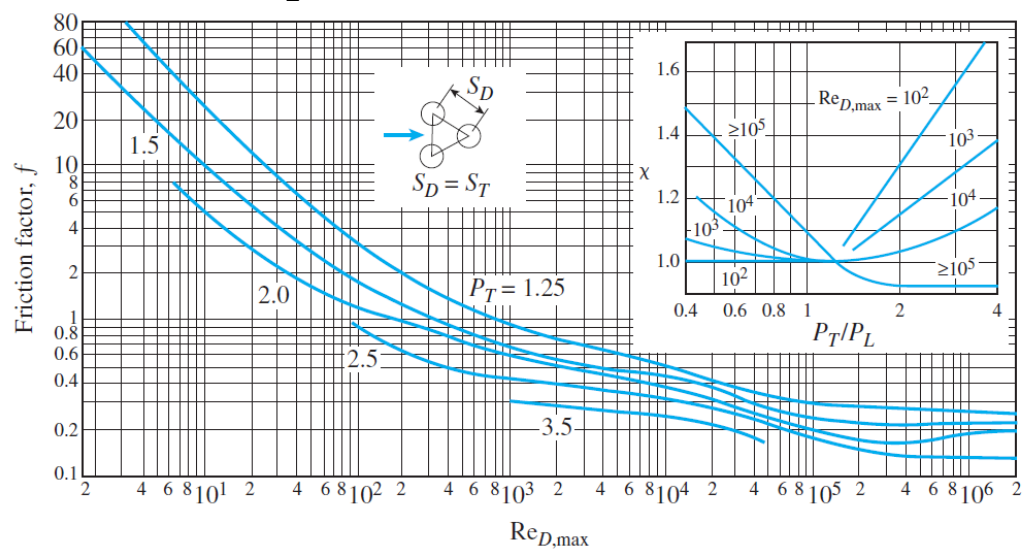

Fig. 2: Friction factor for a staggered array of tubes [14]. 


\section{Results and Discussion}

The current design consists of a bundle of copper tubes with $8 \mathrm{~mm}$ inner diameter with $1 \mathrm{~mm}$ wall thickness and a cylindrical outer steel shell with $70 \mathrm{~mm}$ inner diameter and $5 \mathrm{~mm}$ wall thickness. The bundle of tubes is $180 \mathrm{~mm}$ long and it consists of 36 tubes. The total length of the heat exchanger is $260 \mathrm{~mm}$, including the semi-spherical end caps. It is intended to cool a flow of oil at a flowrate of $1800 \mathrm{~kg} / \mathrm{h}$ that enters from the upper right, using water as coolant at a flowrate of $450 \mathrm{~kg} / \mathrm{h}$ that enters from the lower left (Fig. 3). The inlet temperatures of oil and water are $330^{\circ} \mathrm{K}$ and $300^{\circ} \mathrm{K}$, respectively. Oil has a kinematic viscosity of $20 \mathrm{cSt}$ and density of $890 \mathrm{~kg} / \mathrm{m}^{3}$. Outside surface of shell is assumed to be insulated. Using these data and Eq.s 1-9, one comes up with $R_{\text {in }}=1105, R_{\text {out }}=300, U=377.4 \mathrm{~W} / \mathrm{m}^{2}-C, N T U=0.147, \varepsilon$ $=0.131$, thus $\dot{\boldsymbol{Q}}_{\max }=15,675 \mathrm{~W}$ and $\dot{\boldsymbol{Q}}=2053 \mathrm{~W}$. A friction factor of $f=1.6$ and the factor of $X=1.25$ are adopted using Fig. 2 and a pressure drop of $\triangle P=10,880 \mathrm{~Pa}$ is obtained. The numerical model is validated according to the these calculated values. The first step in using CFD is to construct the geometry (Fig. 4). Owing to the symmetry of the geometry, only half of the domain is studied in order to facilitate the efficient use of computational resources. The geometry of the heat exchanger used for model has 6 parts: Tubes, Shell, Front cap, Back cap, Oil flow domain and Water flow domain. The second step is to construct the mesh for the computational domain. Mesh size and type, thus the number of mesh elements decide both the precision of the results and the execution time. They were optimized by performing a series of trials until the calculations become invariant. The mesh structure shown in Fig. 5 has 2.8 million mesh elements for the half domain.

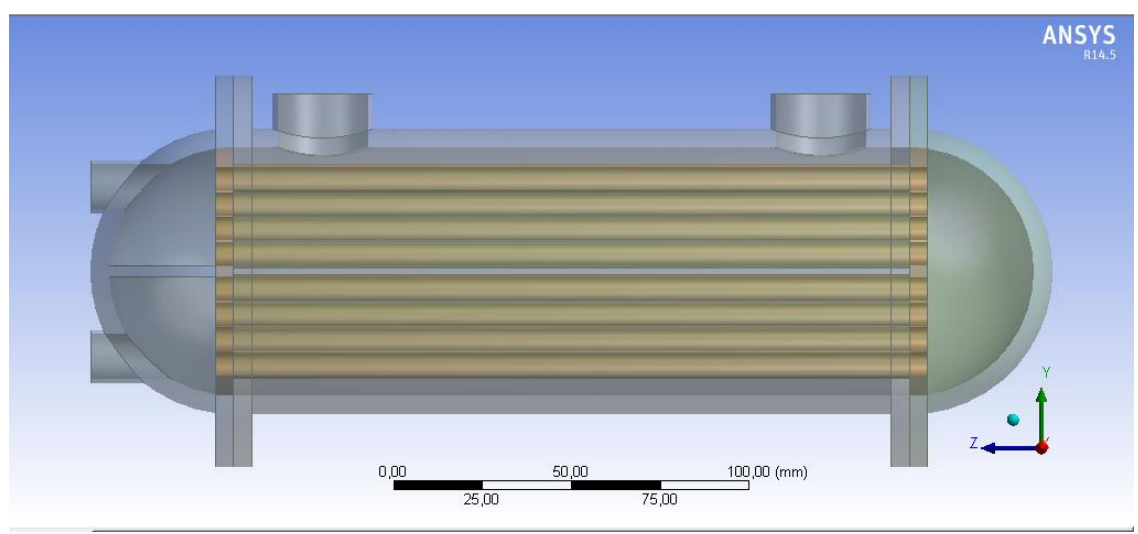

Fig. 3: Layout of the shell tube heat exchanger.

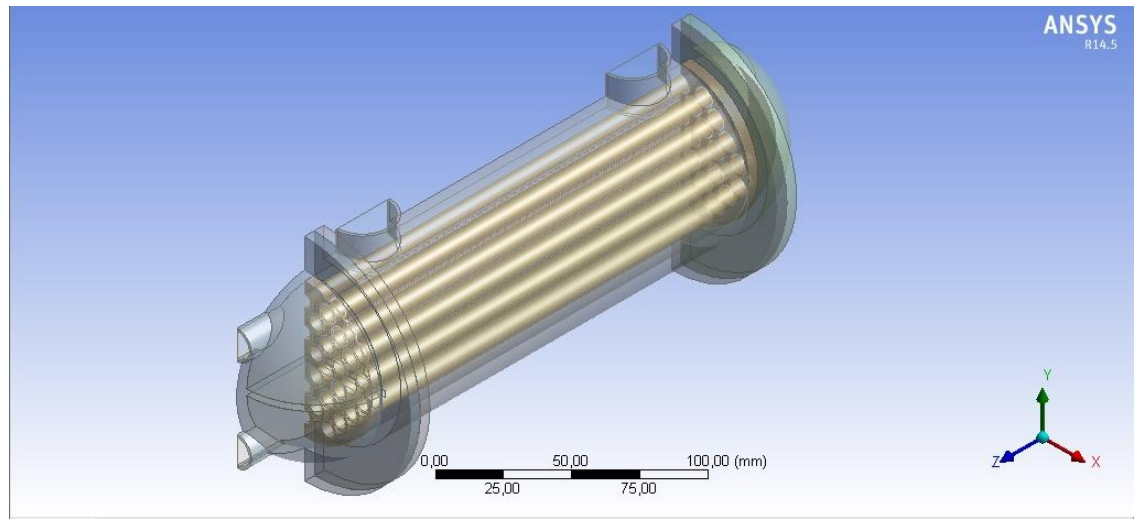

Fig. 4: Geometry of the model in ANSYS Fluent ${ }^{\circ}$. 


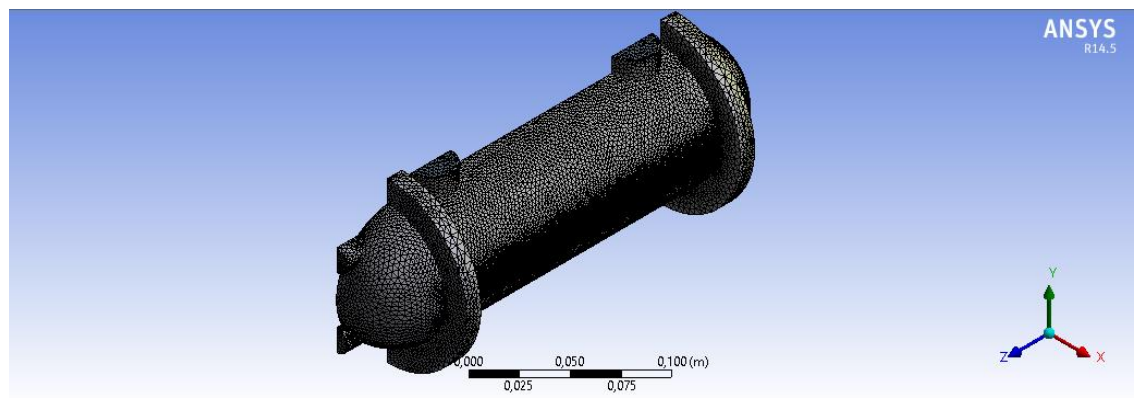

Fig. 5: Mesh of the model in ANSYS Fluent ${ }^{\circledR}$.

Next step is the problem setup which is the most critical one. The pressure-based steady-state solver is chosen. Fluid flow with designated inlet mass flow rates and temperatures considering realizable $k-\varepsilon$ turbulent model coupled with energy equation is the model studied. ANSYS Fluent ${ }^{\oplus}$ is designed to simultaneously solve all three conservation equations, namely conservation of mass, momentum and energy, respectively, as follows, using finite volume formulation:

$$
\begin{gathered}
\vec{\nabla} \cdot(\rho \vec{V})=0 \\
\vec{\nabla} \cdot(\rho \vec{V} \cdot \vec{V})=-\vec{\nabla} p+\vec{\nabla} \cdot(\overline{\bar{\tau}})+\rho \vec{g} \\
\vec{\nabla} \cdot([\vec{V}(\rho E+p)])=\vec{\nabla} \cdot\left[k_{e f f} \vec{\nabla} T-(\overline{\bar{\tau}} \cdot \vec{V})\right]
\end{gathered}
$$

where $\overline{\overline{\boldsymbol{\tau}}}$ represents the stress tensor and $\boldsymbol{k}_{\text {eff }}$ represents the effective thermal conductivity of fluids, both taking into account turbulent effects. Inlet boundary conditions are the aforementioned oil and water flow rates and temperatures. Outlet boundary condition is atmospheric air pressure, or zero gage pressure. Under-relaxation factors are adjusted in order to prevent divergence, even though this procedure slows down the convergence considerably.

Fig.s 6-9 show the results of the model to be validated. The computed heat exchange rate between oil and water being $1961 \mathrm{~W}$, the heat transfer results show that the CFD model has only a difference with analytical results by $4.48 \%$. The computed pressure drop in oil side is $\triangle P=10,275 \mathrm{~Pa}$, representing a $5.56 \%$ difference.

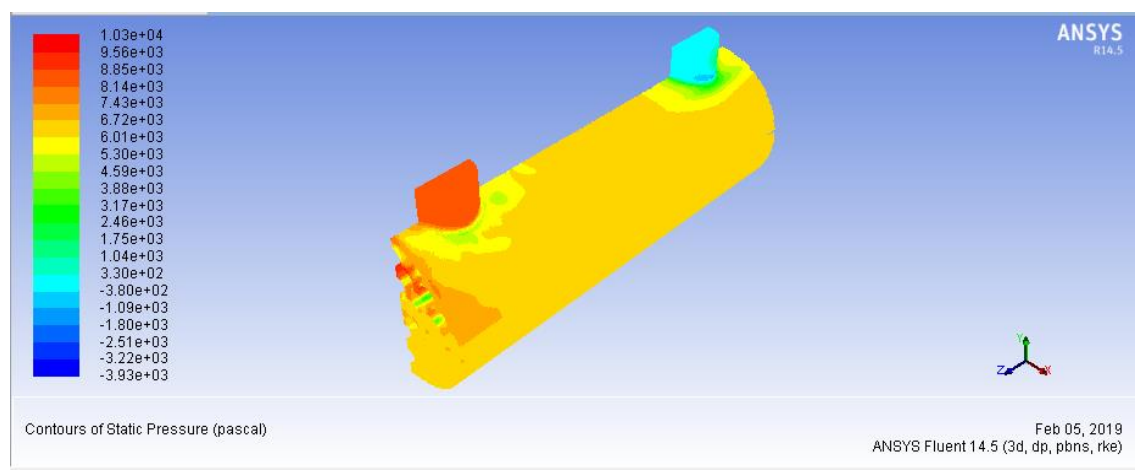

Fig. 6: Pressure distribution in oil side. 


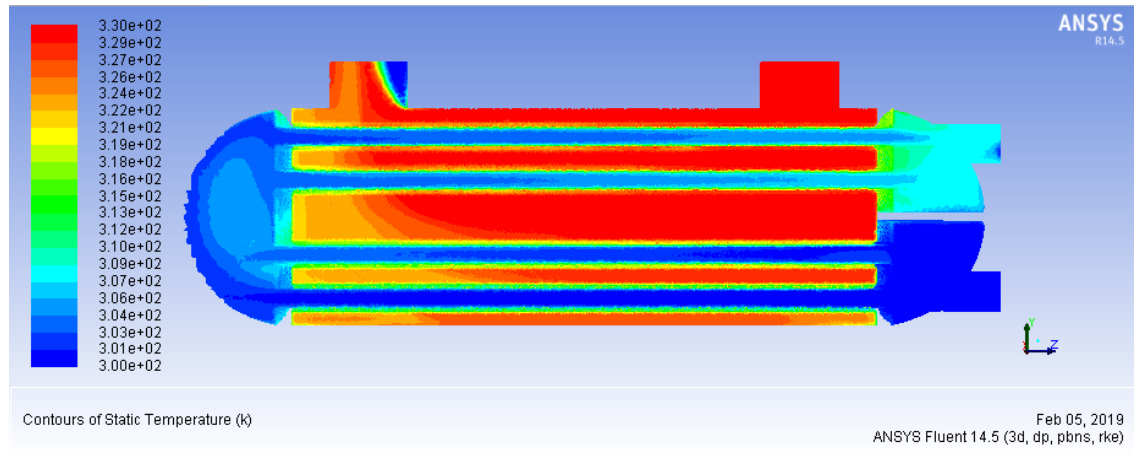

Fig. 7: Temperature distribution in both fluids.

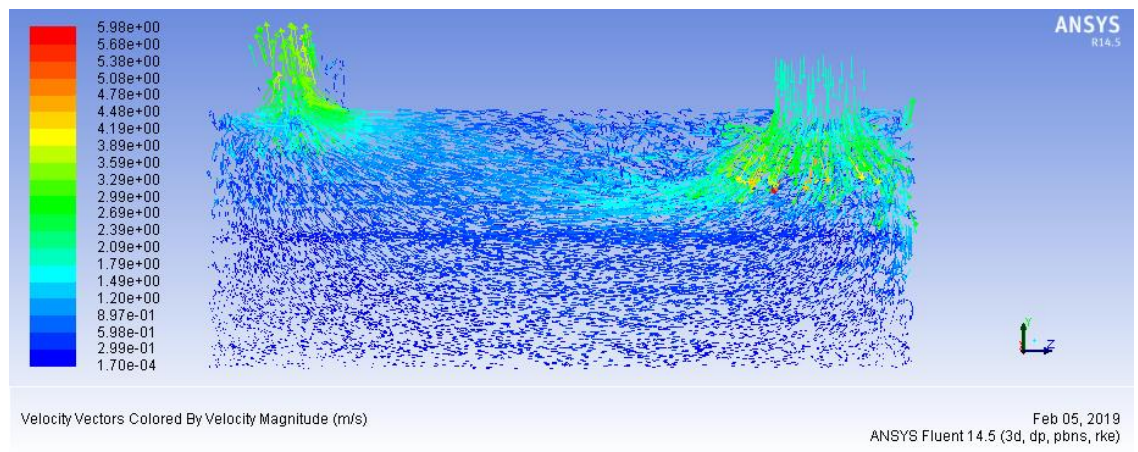

Fig. 8: Velocity vectors in oil side.

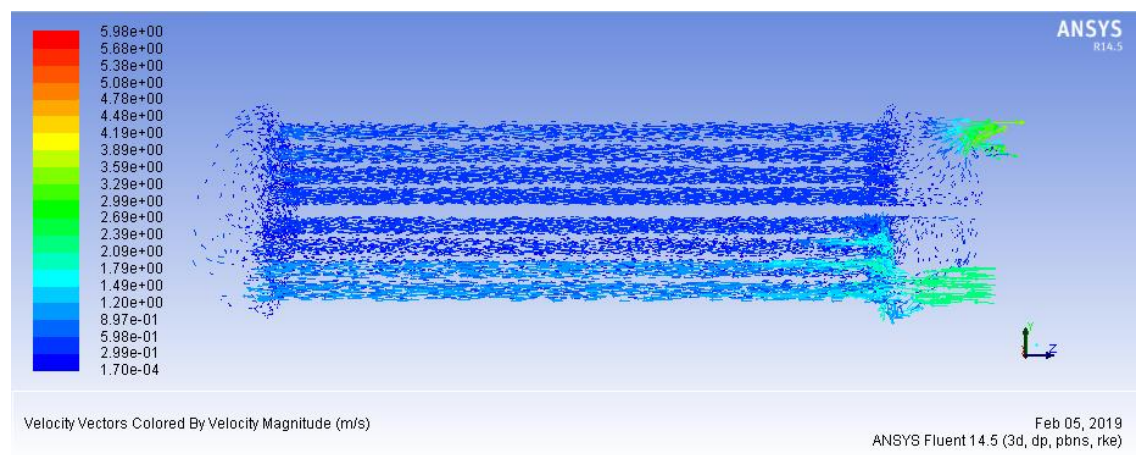

Fig. 9: Velocity vectors in water side.

\section{Conclusion}

In this numerical work, a numerical model for a Shell and Tube heat exchanger is studied, using the commercial software ANSYS Fluent ${ }^{\odot}$. The results compared to those obtained from the calculations have shown that, ANSYS Fluent ${ }^{\odot}$ produces realistic outputs as far as the heat transfer and the hydrodynamics in fluid flow through the Shell and Tube heat exchangers are concerned. It is concluded that, following a preliminary design of a Shell and Tube heat exchanger obtained from the analytical formulas and empirical correlations can be validated using a reliable CFD package such as ANSYS Fluent $^{\odot}$, making it possible to revise the model as needed before a prototype is manufactured and bench tested. A followup to this study may be adding baffles into the oil flow domain in different configurations to enhance the heat transfer, however at a penalty of higher pressure loss, thus higher pumping costs. 


\section{References}

[1] Hajabdollahi, H., Naderi, M., Adimi, S., A comparative study on the shell and tube and gasket-plate heat exchangers: The economic viewpoint, Applied Thermal Engineering 92 (2016) pp. 271-282

[2] Wang, S., Wen, J., Li, Y., An experimental investigation of heat transfer enhancement for a shell-and-tube heat exchanger, Applied Thermal Engineering. vol. 29 (2009) pp. 2433-2438

[3] Parikshit, B., Spandana, K.R., Krishna, V., Seetharam, T.R., Seetharamu, K.N., A simple method to calculate shell side fluid pressure drop in a shell and tube heat exchanger, International Journal of Heat and Mass Transfer. Vol. 84 (2015) pp 700-712

[4] Vera-García, F., García-Cascales, J.R., Gonzálvez-Maciá, J., Cabello, R., Llopis, R., Sanchez, D., Torrella, E., A simplified model for shell-and-tubes heat exchangers: Practical application, Applied Thermal Engineering. Vol. 30 (2010) pp. 1231-1241

[5] Wang, Y., Gu, X., Jin, Z, Wang, K., Characteristics of heat transfer for tube banks in crossflow and its relation with that in shell-and-tube heat exchangers, International Journal of Heat and Mass Transfer. vol. 93 (2016) pp. 584-594.

[6] Fettaka, S., Thibault, J., Gupta; Y., Design of shell-and-tube heat exchangers using multiobjective optimization, International Journal of Heat and Mass Transfer 60 (2013) pp. 343-354.

[7] Costa, A.L.H. and Queiroz, E.M., Design optimization of shell-and-tube heat exchangers, Applied Thermal 28 (2008) pp. 1798-1805.

[8] Raja, R.D., Jhala, R.L., Patel, V., Many-objective optimization of shell and tube heat exchanger, Thermal Science and Engineering Progress 2 (2017) pp. 87-101

[9] Haitao, H., Zhancheng, L., Feilong, Z., Song, C., Guoliang, D., Dawei, Z., Optimization of tube bundle arrangement flooded shell-and-tube evaporator, Energy Procedia 142 (2017) pp. 3799-3804.

[10] Abda, A.A., Kareema, M.Q., Najib,S.Z., Performance analysis of shell and tube heat exchanger: Parametric study, Case Studies in Thermal Engineering 12 (2018) pp. 563-.568

[11] Zhou, G., Zhu, L.Y., Zhu, H., Tu, S., Lei,J., Prediction of temperature distribution in shell-and-tube heat exchangers, Energy Procedia 61 ( 2014 ) pp. $799-802$.

[12] Çengel, Y.A., Heat Transfer: A Practical Approach, $2^{\text {nd }}$ Edition, ISBN-10: 9780072458930, McGraw Hill, 2003.

[13] Žkauskas, J.A., Heat Transfer from Tubes in Crossflow, Advances in Heat Transfer, Volume 18, 1987, Pages 87159.

[14] Çengel, Y.A. and Ghajar, J.A., Heat and mass Transfer: Fundamentals \& Applications, $5^{\text {th }}$ Edition, ISBN 978-0-07339818-1, McGraw Hill, 2015. 